\title{
Organizational Commitment Scale Validation in Tanzanian Context
}

\author{
Dr. Henry Jonathan \\ Human Resource Management Department, Local Government Training Institute, Dodoma, Tanzania \\ P.O BOX 1125, Dodoma, Tanzania
}

\begin{abstract}
The study was designed to investigate Constructs Independence; Normality; Reliability and Validity of Allen and Meyer's Questionnaire (1990) three constructs namely Affective, Normative and Continuous Commitment in Tanzanian data. It was purposively planned and conducted in two separate phases (Study 1 and 2). In study 1; Tanzanian data were tested if Allen and Meyer's Questionnaire (1990) yielded independent constructs. In study 2; tests for validity, reliability and normality levels were primary to the study. All tests came following solid theoretical background pertaining to how Hofstede (2001) cultural dimensions determine response styles, response bias and questionnaire response across cultures. A total of total of 104 and 214 respondents from Tanzanian Councils were involved in study 1 and study 2 respectively. Principal Component Analysis (PCA) indicated Meyers and Allen three scales were component of common constructs. Specified PCA models on the three scales showed significant results indicated by $\mathrm{p}<.05$; and KMO greater than 0.6 . and no loading variable was worse than 0.90. Similarly; Normality Analysis suggested data collected yielded normality; as combined graph produced Skewness (.265); Kurtosis (-1.427) and skewness value range between -.5 and +.5. Also; Cronbach Alpha Analyses indicated; data collected had Cronbach Alpha better than .70 the reliability minimum requirement scores in all three questionnaire constructs. Further; Confirmatory Factors Analysis revealed that Meyers and Allen Questionnaire yielded significant validity using fit indices RMR (Root Mean Square Residual), GFI (Goodness Fit Index), TLI (Tucker-Lewis Index), CFI (Comparative Fit Index) and RMSEA (Root Mean Square Error of Approximation). Thus, it was concluded that Meyers and Allen Questionnaire is reliable and valid for organizational commitment studies in Tanzanian context. Recommendations for reducing or eliminating questionnaire response bias to have better statistical analyses and estimations were included.
\end{abstract}

Keywords: Organizational Commitment; Cultural Dimension; Response Styles; Councils; Tanzania

DOI: $10.7176 /$ RHSS/10-22-09

Publication date: November $30^{\text {th }} 2020$

\section{Introduction}

Knowledge advancement certainly depends significantly on the superiority of the measurement scales utilized in the studies (Kanning \& Hill 2013). Kanning and Hill; suggests; the quality of the measured constructs can be realized; when respective measures are defined in objective, reliable and valid manner. In practice; psychological constructs studies are conducted using questionnaires where targeted respondents are subjected to complete set of questions (Wetzel et al, 2015). Often; questionnaires focus in assessing psychological constructs including but not limited to perceptions of interests, as personality traits, or attitudes. Of course; in obvious ways; constructs in questionnaires are closed and spread in Likert scale responses (Wetzel et al, 2015). Central assumption in utilizing questionnaires; is that participants would be affected by two constructs namely latent trait level and the random error (Paulhus, 1991). However; it is not always practical. Literature indicates; respondents may in addition; be influenced by response bias and cultural background (Paulhus, 1991; Wetzel et al, 2015; Harzing, 2006). Literature in questionnaire responses; reveals cultural dimension (collectivism, uncertainty avoidance, power distance, and masculinity) influences respondents towards some specific response styles (Johnson et al., 2005). Conceivably; cultural background; largely determine response style exhibited in questionnaires (Harzing, 2006). Indeed, variations in constructs rating may be due to cultural background; not items in the questionnaire. That's why some authors devoted researches on the impact of response bias in questionnaire completion. Certainly, cross-cultural studies have identified response styles and bias as the genesis for questionnaire faults that need remedy (Smith, 2004). Reports indicate; in some cultures; respondents may respond to questionnaire with inclination to certain response style (Harumi, 2011). Thus, differences in questionnaire results may not necessarily be due to questionnaire items; but response bias (Smith, 2004). Consequently, generalization accruing from questionnaires that overlooked cultural differences and response bias; may produce wrong findings for practice consumption.

In research realm; Organizational commitment constructs (Affective; Normative and Continuous) have received extensive range of considerations in organizational psychological studies for quite several years (Gutierrez et al, 2012). As such, researches on organizational commitment are leading in the literature of Human Resource Management (Maqsood et al, 2012). As a pivotal staff attitude, organizational commitment is considered as vital construct of work behavior across work places (Riketta, 2002). Researches indicate; organizational commitment is fundamental construct to measure among public servants; for example (Bogler \& Somech, 2004). 
Several researchers have designed questionnaires for collecting Organizational Commitment data by testing validity and reliability before actual use (Mugizi et al, 2016). For instance; Mowday et al. (1979) \& Cook \& Wall (1980). In this trend, Allen and Meyer (1990) questionnaire have engrossed great attention of researchers in collecting data for organizational commitment (Mugizi et al, 2016). Principally, Allen and Meyer (1990) questionnaire were developed to measure organizational commitment basing on three facets namely of affective (AC), continuance (CC) and normative (NC) commitment. It was tested (in western culture) against validity and reliability using manufacturing and University employees working on full time category (Allen \& Meyer, 1990). Though, Allen and Meyer reported affective (AC), continuance (CC) and normative (NC) commitment were valid, reliable and independent; affective (AC) and normative (NC) were related (Meyer \& Allen, 2004) either.

On validation rapports; Allen and Meyer's Questionnaire (1990) has received significant Psychometric Properties scrutiny across western and non-western cultures. For example, Pakistan, Abbas \& Khanam (2013); Malaysia, Karim \& Noor (2006); Mexico, Norma \& Francisco (2007); Uganda, Wilson et al, (2016) and Indonesia, Suryani \& Tentama (2020). Findings out of the previous study reviewed; produced inconsistent findings and in some cases contradictory in nature. For example; some findings suggested Allen and Meyer's Questionnaire Psychometric Properties matches local data and thus can be adopted in organizational commitment studies (Eg; Abbas \& Khanam (2013); Wilson et al, (2016); Suryani \& Tentama (2020)). Conversely, some findings indicated the scale produces poor Psychometric Properties where adjustment prior usage is of essence (Eg; Solinger et al, (2008); Maqsood et al, (2012) and Pais et al, (2014)). Yet, though, there are literature indications of vigorous validity and reliability studies (Allen \& Meyer's Questionnaire (1990)) conducted in western cultures; few (if any) have reported validity and reliability studies in African cultures (Jaros, 2007). This study, therefore, build significance on four evidences drawn from the literature: - One: Allen and Meyer's Questionnaire (1990) was developed and validated in western cultures where according to Hofstede, (2001) generally falls under individualism while Tanzanian falls under collectivism. Two: Cultural differences influences response styles on questionnaires and that response in individualism (western) may vary with that in collectivism (Tanzania). Three: Psychometric Properties Findings on Allen and Meyer's Questionnaire (1990) shown inconsistent and contradictory results which calls for more researches in the area. Four: There is draught of Allen and Meyer's Questionnaire (1990) validity and reliability studies in African context; Tanzania in particular. On this background; this study is designed to address the following objectives:

1. To examine whether or not; Allen and Meyer's Questionnaire (1990) namely Affective, Normative and Continuous Commitment are independent constructs using Tanzanian data

2. To investigate the validity, reliability and normality levels of Allen and Meyer's Questionnaire (1990) using Tanzanian data

This study was certainly planned and conducted in two separate sessions namely Study 1 and Study 2 . While Study 1 was designed to focus on testing as to whether Allen and Meyer's Questionnaire (1990) had independent constructs; Study 2 focused in testing validity, reliability and normality levels. The two studies (Study $1 \& 2$ ) were preceded by solid literature review that uncovers three information categories. One: How cultural dimensions namely; collectivism, uncertainty avoidance, power distance, and masculinity determine response styles and response bias. Two: How response styles and response bias are likely source of error in standardized questionnaires affecting questionnaire validity and reliability. Three: How available literature exhibit gaps to be addressed. Indeed; reviewed literature produced inconsistent findings and in some cases contradictory in nature.

\section{Theoretical Foundation}

\subsection{Questionnaire, Culture and Response Bias}

Many psychological variable investigations are conducted using questionnaires where targeted participants are requested to complete set of questions (Wetzel et al, 2015). Customarily, items in questionnaire focus in assessing psychological facets including but not limited to perceptions of interests, as personality traits, or attitudes. According to Wetzel et al, items in the questionnaires are closed and spread in Likert scale responses. Fundamental assumption when questionnaire is employed; is that respondents are affected by two constructs namely latent trait level and the random error (Paulhus, 1991). This is valid if it is certain; there is no other orderly effect on responding to questionnaire. Researches indicate; this is not practical; as there are other factors that affect items scores in the questionnaires for example; response bias and cultural context (Paulhus, 1991; Wetzel et al, 2015). This is supported by authors who suggests; cultural background influence respondent's preferences to some response styles than others (Harzing, 2006). Consequently; it is presumed; cultural dimensions namely; collectivism, uncertainty avoidance, power distance, and masculinity determine response styles (Harumi, 2011). Hofstede (2011) offered definitions to these cultural dimensions as follows: - Power Distance (PD): The degree to which citizens in the society agree and expect uneven power distribution. Society high in PD agrees ranked power distribution and admits their classification in the hierarch (Hofstede et al, 2010). Conversely, Society low in PD expect power to be shared and will never welcome uneven power distribution. Practically, societies high in PD expect guidance before engaging in any activity (Hofstede, 2001). Thus, they rarely initiate any deed; unless 
directives are certain. Individualism VS Collectivism: Refers the extent to which people in the society are cohesive in the groups. Society high in individualism; display puny interpersonal connections and exhibit lesser obligations to others deeds and respective outcomes (Hofstede, 2001). Bonds between society members is weak and every member is obliged to take care of own family members; not the larger group (Hofstede, 2001). Contrariwise, people high in collectivism; tend to be loyal to the group; and defend group interests. Individualism is high in industrialized and western societies and less in developing countries (Hofstede et al. 2010). Masculinity VS Femininity: Describe the manner roles are distributed between men and women. Society high in masculine; show lesser roles overlap between men and women. Lesser roles overlap facilitates men to act assertively (Hofstede et al., 1998). On the other hand; society high in feminine; show boundless deed of roles between gender. The society place considerable respect on relationships with people in hierarch or people they work with (Hofstede et al, 2011). Masculinity is high in countries such as Japan, German and Mexico; Low in Western countries, Nordic countries and moderately in France, Spain, Portugal, Chile, Korea and Thailand (Hofstede et al, 2010). Uncertainty Avoidance: Refers the extent to which members possess thrust in tolerating ambiguities. In other words; it refers the degree to which cultural dimensions nurture society feel uncomfortable or comfortable in in unstructured state of affairs (Hofstede et al., 1998). Society high in Uncertainty Avoidance; would make sure endeavors are foreseeable and manageable as possible. Contrary, society low in Uncertainty Avoidance; are greatly open and inclusive (Hofstede et al, 2011). Uncertainty Avoidance; is high in European countries, Latin countries, Japan and German; Low in English speaking, Nordic and Chinese countries (Hofstede, 2010)

Alternatively, Response bias is defined as orderly deviation of self-reported respondent rates from true rates (Bogner et al, 2016). It is also understood to mean, inclination to some response styles based on constructs format as opposed to factual opinion or emotional state (Fischer, 2004). According to Bogner et al, response bias is likely source of error in standardized questionnaires affecting questionnaire validity. Researchers utilizing questionnaires as data collecting tools; depend on truth responses provided by respondents to arrive at conclusions (Mortel, 2015). Thus, validity of the questionnaire constructs is of essence. Questionnaires are valid if they correctly measure what is expected to measure (Beanland et al 1999). Nevertheless; it is known; about $10 \%$ to $75 \%$ of the variations in respondents' responses may be described by response bias (Nederhof, 1985). Traditionally, response bias confounds constructs associations through defeating constructs associations or creating false associations between constructs of interest (King \& Brunner 2000). There are three common types of response bias namely Acquiescence Responding Style (ARS), Extreme Responding Style (ERS) and Midpoint Responding Style (MRS). The following review (response bias) focuses in showing response variations across cultures; with potential effect to questionnaires validity.

\subsubsection{Acquiescence Response Bias}

Acquiescence is identified as one among response biases which are sovereign of questionnaire questions content (Krosnick, 1991). Questionnaire respondents with acquiescence bias agree to questions regardless of the content. Often, their inclinations preferences are on agree, true or yes responses (Krosnick, 1999). In particular, this type of response bias produces systematic distortions; that affect questionnaire validity either (Billiet \& Davidov, 2008). Literature have conflicting finding as far as response style and cultural differences is concerned. For instance; In USA, individuals whose English is their second language like Mexican Americans are more likely to acquiesce; than counterpart whose English language is mother tongue (Ross \& Mirowsky 1984). Conversely, other researchers indicated no significant acquiesce differences to report between blacks and whites in cross cultures (Bachman \& O'Malley 1984). For instance; comparison study on African Americans, Hispanics and White Americans found Acquiescence Response Style (ARS) is more likely in African Americans and Hispanics than White Americans (Marin et al., 1992). Again, the Acquiescence Response Style (ARS) likelihood; is found to exhibit more in individualistic respondents such as western Europe than less individualistic respondents like Mediterranean nations (Verhallen et al, 2004). Yet, Acquiescence Response Style (ARS) is found to feature more in blacks and whites originating from southern states in USA. Acquiescence Response Style (ARS) also, seems to be lower in states higher in Hofstede's dimensions namely individualism, uncertainty avoidance, masculinity, and power distance (Johnson et al. 2005). More specifically; respondents in individualistic states are more likely to acquiesce than respondents of collectivistic origin. For instance; Individualistic and collectivistic state comparison study found Philippines (collectivist) respondents to be significantly related to Acquiescence Response compared to USA which is individualist oriented (Grimm \& Church 1999). That's how study findings interlock and conflict each other.

In a unique fashion; Smith (2004) tried to examine whether Acquiescence Response Style (ARS) was associated with cultural orientations as well. While most studies perceived Acquiescence Response Style (ARS) as key of questionnaire completion error; Smith tested contribution of type of construct the questionnaire measure. He found; Acquiescence Response Style (ARS) was likely on questionnaires that focused to measure personal based values. The more respondents were asked on constructs of personal values (such as attitudes, beliefs, and behaviors); the more the questionnaire produced significant estimates of Acquiescence Response bias. It can therefore be concluded that; Acquiescence Response Style (ARS) vary across cultural States and thus response 
level on the same questionnaire may vary across cultural States. Further; States stronger in Hofstede's collectivism reported stronger Acquiescence Response in general. Though, Acquiescence Response Style (ARS) and cultural orientation association was found; there is lack of consistency and conflicting findings have been noted. Thus, more investigation in the area is imperative.

\subsubsection{Extreme Response Bias}

Extreme Response Style (ERS) is defined as the inclination to select questionnaire items located in extreme ends of the continuum such as strongly agree or strongly disagree in likert scale (Chun et al. 1974). It refers respondents who consistently select endpoints scale perception rates in questionnaire (Dolnicar \& Grun, 2007). Earlier studies have reported Extreme Response bias consequences, if strategies to correct are ignored. For example; Chun et al. (1974) reported three consequences including: - One: Variations in Extreme responses may result in mean variation that researcher cannot interpret. Two: Variations in Extreme responses may impact constructs correlations and inflate internal consistency index; which produce false internal consistency. Three: Variations in Extreme responses may impact constructs correlations, which may in turn affect outcome analyses such as factor analyses. In specific terms; if happens, Extreme Response bias inflate reliability and shrink validity (Clarke, 2001). Consequently, it twists frequency distribution to extremes low or high. In turn, it raises standard deviation and cut correlation. Every so often, Extreme Response bias, in questionnaires produces false responses that misrepresent covariance-based analyses (Rossi et al, 2001). In some cases; if questionnaire respondents exhibit Extreme Response bias; regression analysis and distance-based analysis will be misrepresented (Greenleaf, 1992)

Researchers have also examined cross-cultural variations in response styles using questionnaires as data collecting instrument. The focus in these studies was primarily to check, if at all, cultural differences in Extreme Response Style could produce confounding outcome. For instance; Clarke (2000) conducted a comparison study to explore Extreme Response variations between non-Hispanic White students in USA VS students in Mexico; Black VS White students in USA and students in Australia VS France. Clarke reported; Mexican students registered higher levels of Extreme Response Style as compared to non-Hispanic Whites across all response formats. Further, the study found higher Extreme Response Style in Black students than Whites. On French and Australian students; the earlier reported higher Extreme Response Style than the latter. Technically, this finding represents traditional reports that compared Extreme Response Style in cultures (Harumi, 2011). According to Harumi, other research category; captures features of the cultures associated with Extreme Response Style. Van Herk et al. (2004) investigated extreme responses share selected in questionnaires constructs involving six European countries. In female category; the study found Greek participants were higher in Extreme Response Style mean than all European countries in the study. Hitherto; male counterpart; Italy and Spain reported higher Extreme Response Style mean than France, Germany, and the United Kingdom. In aggregate; findings suggested Mediterranean (Greece, Spain, and Italy) respondents were high in Extreme Response Style than Northwestern European (France, United Kingdom, and Germany) respondents. On cultural lens; it was deduced; collectivism respondents were high in Extreme Response Style than individualistic respondents. Alternatively, Johnson et al. (2005) explored cultural - Extreme Response Style association in nineteen different States. In addition, the impact of individual characteristics such as gender, age, length of employment and per capita GNP on Extreme Response Style variations were studied. Study expectations were; high Extreme Response Style would feature in countries higher in individualist, masculine, and high in power distance. However, actual findings suggested cultural Extreme Response Style association was not significant. Stating otherwise, association between Extreme Response Style and cultural orientation (individualism or uncertainty avoidance) was not significant. But Extreme Response Style was related to cultural orientation (power distance and masculinity) in positive fashion instead.

On the other hand; Harzing (2006) studied Extreme Response Style association with several factors including cultural orientations and language of the survey. Harzing wanted to test hypothesis that; Extreme Response Style levels would be higher if mother tongue language is employed in the questionnaire. Findings were in affirmative in language category. Response Style levels were high when respondents were subjected to rate questionnaire in mother tongue language. On cultural orientation lens, the study found Extreme Response Style - cultural orientation was significant. Similarly; Lamm and Keller (2007) conducted a comparative study on Extreme Response Style variations in completing questionnaires between mothers. Intracultural variation - Extreme Response Style association was also studied. The study found high Extreme Response Style in interdependent culture mothers (Cameroonian and Gujarati) in one hand. Mothers from autonomous-relatedness culture (urban Indian, Chinese, Mexican, and Costa Rican) were also higher in Extreme Response Style than independent cultural orientations mothers (German, Euro-American, and Greek). Despite notice of some consistent findings; the review noted inconsistent or contradictory results as well. For example; Chen et al. (1995) conducted a comparative study on mean number of extreme responses of questionnaire respondents in individualistic culture (USA and Canada) and collectivist culture (China and Japan). Findings indicated the association between high scores in individualism scale and Extreme Response Style was significant. Moreover, USA respondents were more likely to rate constructs to extreme points than Japanese and Chinese counterpart. Though, USA respondents were also more likely to rate constructs in extreme points than Canadian respondents. Yet, the study found Canadian, Japanese, and Chinese 
respondents did not vary in Extreme Response Style. It is therefore rational to conclude that; there are significant variations in Extreme Response Style when questionnaires are completed with different cultural background respondents. Further, Extreme Response Style variations in some cultures (individualistic and collectivistic) did not produce steady and consistent output. Findings concluded some cultures (individualistic and collectivistic) could not account for Extreme Response Style across cultures. Again, Cultural characteristics associated with determination of Extreme Response Style were not consistent as well. This calls for further studies in an attempt to draw steady and consistent findings on cultural characteristics which account for Extreme Response Style variations.

\subsubsection{Midpoint Response Bias}

Midpoint Responding Style (MRS), refers an inclination to rate constructs in questionnaires on neutral classes (Harumi, 2011). It is characterized by bipolar instruments in which participants are not obliged to select precise path in the scale (Anna, 2017). According to Anna; Midpoint response options are provided either implicit or explicit. In explicit terms; midpoint response is tagged as "neither X nor Y. However, there is no need of indications when it comes to implicit category; it speaks by itself. Literature provides two competing arguments on midpoint provisions in the questionnaire. First argument is that providing midpoint options; surge the risk of study satisficing (Kulas \& Stachowski, 2009). Second argument is that; removing midpoint provisions; compel respondents to select options against real attitudinal situation (Sturgis et al. 2014). Nevertheless, midpoint provision in the questionnaire may be regarded as utmost typical and can be used to make study conclusions (Tourangeau et al, 2004). Some studies reported; midpoint response provisions may likely surge acquiescence and lowers the inclination in the extreme responding direction (Weijters et al.2010). Prior findings suggested; if given neutral rating options; respondents are more likely to rate constructs closer to neutral scores (Si \& Cullen, 1998). According to Harumi (2011); cross-cultural study findings are grossly affected; if some respondents engage; in any way; neutral inclination in completing questionnaires. Reports on meaningfully variations in Midpoint Responding Style across cultures have been registered. Taking an example; Chen et al. (1995) surveyed midpoint response variations for collectivistic respondents (Japan and China) and individualistic respondents (Canada and USA). Basically, they compared midpoint responses mean scores in likert scale. The questionnaire had midpoints response ranging from "somewhat important", "average", or "neither agree nor disagree. Final results indicated collectivistic respondents selected more midpoints responses than any individualistic respondents. Further, individualistic respondents indicated negative association to midpoint responses. In the same vein; Si \& Cullen (1998); tested hypothesis that "The effect of Confucian philosophy would lead collectivist questionnaire respondents (China, Japan, and Hong Kong) prefer midpoint scores than individualistic respondents (USA, Germany, and UK)". The focus was to compare midpoint variation preferences in East Asia respondents and western respondents on likert scaled questionnaire. The study found; collectivist respondents exhibited midpoint response than individualistic respondents did. Uniquely, collectivist respondents were higher in midpoint response when odd number was used in the questionnaire; but not even number. A conclusion was made that; rooting out neutral response options; may be used to curb midpoint response inclinations. Moreover; Hamamura et al. (2007) compared Midpoint Response Style (MRS) between collectivist respondents living in individualistic and; European respondents living in USA and Canada. Results reported collectivist respondents living in individualistic cultures opted for moderate response in the questionnaires, than Europe respondents in USA and Canada did. Despite change of locality; still collectivist respondents consistently went for middle of the scale options.

Although there are some consistency findings; reports recorded inconsistency findings as well. For instance; Grimm \& Church (1999); examined midpoint response disparities; between Philippines respondents (Collectivistic) and USA respondents (Individualistic). Comparison was made on the respondents' midpoint preferences in the questionnaire's completion. Unexpectedly, results did not support hypothesis "individualistic response is inversely proportional to midpoint questionnaire response". In addition, the study found, no meaningful midpoint response variations between individualistic and collectivistic respondents in the study. Thus, individualistic and collectivistic cultural criteria did not explain midpoint response variations in the questionnaire completed. Likewise, Harzing (2006) investigated several questions in an attempt to find out midpoint response across cultures. One: Find out the association nature of the cultural dimensions (power distance, collectivism, uncertainty avoidance) and Midpoint Response Style (MRS). Two: Determine the role that gender and language plays in midpoint variations across cultures. Results revealed; Cultural constructs namely power distance and collectivism was inversely associated with Midpoint Response Style (MRS). Also; Cultural constructs (uncertainty avoidance) were inversely associated with Midpoint Response Style (MRS). Again, Cultural constructs (power distance, collectivism, uncertainty avoidance); were not, in any way, associated with Midpoint Response Style (MRS). In language relations; Harzing found significant higher Midpoint Response Style when respondents subjected to questionnaires with non-native language. Though, females also reported significant higher Midpoint Response Style than counterpart males. It is objective to conclude; in Midpoint Responding Style (MRS) footings; there is no stable conclusions and more studies are open. 


\subsection{Previous Validation}

Relatively, good numbers of researchers have validated Allen and Meyer's Organizational Commitment Scale in various cultural contexts so far. Certainly, some findings have supported the three constructs of the Allen and Meyer's Organizational Commitment Scale for independence, reliability and validity. For example, Abbas \& Khanam (2013); Karim \& Noor (2006); Norma \& Francisco (2007); Wilson et al, (2016) and Suryani \& Fatwa (2020) just to name those reviewed. Karim \& Noor (2006); studied the adoption of Allen and Meyers' Organizational Commitment Scale psychometric properties using Malaysian respondents. Utilizing parametric statistics methods; they tested construct validity and internal consistency of Affective, Continuous and Normative Commitment constructs. Findings of the three constructs demonstrated construct validity and internal consistency beyond cat-off points; indicating it could be adopted for use in Malaysian Library staff. Thus, conclusion was made that; Allen and Meyer' three construct scale could be adopted in international localities with varying cultures. In the same vein; Norma \& Francisco (2007) examined as to whether Allen and Meyers' Organizational Commitment Scale could be used to assess the purported three constructs namely Affective, Continuous and Normative. Employing rigorous statistical approach; they tested if the scale matches Mexican employees. Exploratory Factor Analyses, Reliability Analyses and Concurrent Validity Analyses suggested results above cutoff criteria too. Hence, conclusion was drawn that; three dimensions in Allen and Meyers' Organizational Commitment Scale may be an option in assessing commitment level in Mexican working environment. Equally; Abbas \& Khanam (2013) validated Allen and Meyers' Organizational Commitment Scale in Pakistan cultural context and tested whether psychometric properties of the scale offer valid and reliable measure. Employing basic parametric statistics techniques, Abbas \& Khanam found Allen and Meyers' Organizational Commitment Scale was reliable and valid scale of assessing organizational commitment in Pakistan cultural context. Psychometric properties result of the three commitment dimensions namely Affective, Continuous and Normative suggested the scale could be used to assess employee commitment in Pakistan cultural context. In the same way, Wilson et al, (2016) examined the validity and reliability of the Allen and Meyer's Organizational Commitment Scale (1990) in Uganda, using employees in Universities. Specifically; they wanted to test validity and reliability of the scale using Ugandan data in one hand. Then, test if at all; the three construct of the scale forms independent constructs using Ugandan data. Confirmatory Factor Analysis (CFA) suggested validities and reliabilities of the affirmative, normative and continuous commitment scale were reasonable. Further; Exploratory Factor Analysis (EFA) indicated affirmative, normative and continuous commitment scale were thorough when applied in Ugandan data. Conclusions were that; researchers in Uganda may use Allen and Meyer's Organizational Commitment Scale (1990) as it fits validity and reliability minimum requirements. Recently, Suryani \& Fatwa (2020) investigated Allen and Meyer's affirmative, normative and continuous commitment construct validity and reliability in Indonesia. In addition; they assessed components purported to form Allen and Meyer's constructs using samples in Universities. Confirmatory Factor Analysis (CFA) proposed construct validity for Allen and Meyer's affirmative, normative and continuous commitment produced meaningful properties. Comparison analysis of the three constructs showed affective leading by 0.946 loading factor; while the lowest was continuance at the tune of 0.647. Cronbach's Alpha Analysis output indicated the three scales produced sound internal consistency that was adequately above the minimum level. Thus, Allen and Meyer's commitment scale is an acceptable scale in Indonesian data as it yielded compatible properties.

Though some literatures have consistently reported sound Allen and Meyer's scale Psychometric Properties in various cultures; some literature reported inconsistency findings either. For example; Maqsood et al, (2012) examined the theoretical structure of Allen and Meyers' Organizational Commitment Scale in Pakistan's higher learning institutions. Utilizing parametric statistical methods, Maqsood et al, found inconsistency in the application of the scale in Pakistan. Confirmatory factor analysis suggested weak association with other items. Further, the utility of bi-dimensionality of Continuous Commitment Construct is questionable. Thus, proposal was made to refine the construct so that it matches Pakistan context. This conflicts Abbas \& Khanam (2013) findings that proposed Allen and Meyers' Organizational Commitment entire Scale matched Pakistan data. Equally, making use of attitude- behavior model as suggested by Eagly and Chaiken (1993); Solinger et al, proposed that Allen and Meyers' Organizational Commitment Scale actually merges vitally different attitudinal phenomena. Thus, lacks essential eligibility criteria as general organizational commitment model. In other words, it endures a conceptual inconsistency and so suffers from unequivocal empirical support. In addition; Pais et al, (2014) validated Allen and Meyers' Organizational Commitment Scale in Portugal call centers sample. Collected data on three Allen and Meyers' constructs were subjected into data analyses techniques including Confirmatory and Exploratory factor analysis. Though in general terms conducted analyses relatively reproduced original structure proposed by Allen and Meyers; some discrepancy was noted in respective validation. That is; assessment of goodness of fit suggested the fit was good; however, this came after eliminating some items and for all indices on account of residual variability of a set of items. This indicated there were percentages of variability that the model could not capture and explain. Proposal was made for future detailed research as it has stake on the general use of the scale. In summary; some literature consistently indicated Allen and Meyers' Organizational Commitment Scale exhibits 
sound psychometric properties across cultures. However; there are evidences that; in some cultures; it lacks command in validity and reliability using local data. Such evidences conflicts scale theoretical background and suggestions of scale generalization across cultures. It is important; therefore; more investigations are extended to Allen and Meyers' (1990) scale in non-western cultures.

\section{Methodology}

\subsection{Study 1}

General objective of the study 1 was to screen Tanzanian data collected by Allen and Meyer's Organizational Commitment Questionnaire and establish if they were component of common constructs. Specific Objectives of the study 1 was three-fold: - One: Extract the most fundamental information from the data set. Two: Compress the size of the data bey retaining fundamental information only. Three: Analyze the structure of the observations and the variables. In the course of achieving these objectives; Principal Component Analysis (PCA) was used to compute new variables namely principle components found as linear combinations of the original variables (Abdi \& Williams, 2010).

\subsubsection{Approach}

Research approach refers the manner a specific research is planned and got implemented (Stets, 1997) basing on research objectives. It is the programming of the research procedures towards research objective achievement (Stets, 2003). Other researchers describe it as the road map showing trends in which the research will be implemented (Burke, 1991). This study was wholly quantitative that utilized closed ended questionnaires to collect data. Several reasons motivated the use of quantitative approach. Grand reason to mention here; included the nature and design of the study itself. The study involved vigorous analysis of statistical techniques; that fits well in quantitative analysis. Supporters of quantitative approach to research argue (quantitative approach strength) on its ability to explain and predict constructs under the study which can be generalized to the larger population (Leedy $\&$ Ormrod, 2010). Quantitative approach offers confidence on not only reliability of collected data but also validity of the gathered data (Pallant, 2005). In fact; these are some of the reasons, quantitative approach was opted in the study.

\subsubsection{Population and Sample}

Population to this study included employees in Tanzanian District councils (working at headquarters) who hold "officer" cadre level in their positions. This means, the study automatically excluded operational staff and other supportive staff whose rank is far from being an officer and work periphery to the headquarters. The reasons for involving this cadre in the study; stem from the fact that; they are the most involved in performance appraisal practice in one hand (Thoits, 1991). Purposive sampling was opted in selecting respondents. Participants to the study were selected on the basis of particular characteristics or identified variable (Iqbal, 2013). This study become purposive; in that; certain employees were chosen (officers) to participate. Available officers were counted to be 377 in total. 48 out of 377 were officers with supervisory responsibilities and 329 were non-supervisors. The study employed Yamane (1967) formula to determine the actual sample size of the study. The basis for selecting this formula includes its simplicity, incorporation of precision level and confidence level in a given population. In mathematical terms the formula can be depicted as: -

$$
\boldsymbol{n}=\frac{\mathrm{N}}{1+\mathrm{N}\left(\mathrm{e}^{2}\right)} \quad \boldsymbol{n}=\frac{377}{1+377\left(0.05^{2}\right)} \quad=194 \text { respondents }
$$

Where $\mathrm{n}=$ study sample; $\mathrm{N}=$ Population of the study; $1=$ constant; $\mathrm{e}=$ level of precision at $95 \%$ confidence level. Thus, it was expected to have 194 officers in the study

\subsubsection{Data collection}

Data were collected using Allen and Meyer's Organizational Commitment Questionnaire (1990). Basically, it measures the extent to which employees are prepared to leave or stay in three subscales namely affirmative, normative and continuous commitment. The questionnaire is made up of three sub-scales namely Affective Commitment; Normative Commitment and Continuous Commitment

\section{Affective Commitment Measurement:}

Affective commitment refers staff association with, participation in and psychological attachment to a corporate accruing from individual desire (Allen \& Meyer, 1997). It also covers the degree of poignant attachment an employee has to the corporate organization (Rashid et al, 2003). Thus, it has to do with the extent to which an employee is emotionally attached to employee corporate through emotions such as loyalty, affection, worth, belongingness, pleasure and countless more (Jaros, 1993). Suffice here to say, staff with affective commitment are characterized by desire to identify themselves with an organizational goals and values (McCaul et al., 1995). So, they stay because they genuinely want to. Affective scale had six (6) items to be completed by respondents. Example items in the questionnaire included..."I would be happy to spend the rest of my career with the organization"....."I really feel as if the organization's problems are my own"......"I do not really feel part of a family at my organization (R)"..... Perception of affective commitment was measured in five scale represented by $1=$ Strongly Disagree; $2=$ Disagree; $3=$ Undecided; $4=$ Agree and $5=$ Strongly Agree 


\section{Normative Commitment Measurement:}

Normative commitment refers to the staff mind-set of obligation and compulsion to stay with the employing organization (Allen and Meyer, 1990). It is all about the extent to which staff feel they should stay with employing organization. Employees acquire feelings that quitting have terrible consequences, and feel a sense of fault on the likelihood of quitting. Normatively committed employees operate in perceived compulsion to stay (Meyer \& Herscovitch, 2001). Thus, employees opt to stay because they ought to do so. Normative commitment Questionnaire had six (6) items. Example items in the questionnaire included..."Even if to my advantage, I do not feel it is right to leave the organization"....."I would feel guilty if I left the organization right now"... "The organization deserves my loyalty".... Perception of normative commitment was measured in five scale represented by $1=$ Strongly Disagree; $2=$ Disagree; $3=$ Undecided; 4= Agree and 5= Strongly Agree

\section{Continuous Commitment Measurement:}

Refers the extent to which an employee is aware of the cost coupled with quitting the employing organization (Allen \& Meyer, 1997). Normally, the higher the feasible alternatives an employee has; the poor continuous commitment become. It sits proper to suggest, employees become committed, under continuous dimensions, simply because of apparent cost of faulting to do so (Meyer \& Herscovitch, 2001). Fundamental reasons for continuous commitment hinges on individual need to stay with the corporate organization (Rashid et al, 2003). According to Rashid et al, feasible reasons for needing to stay include but not limited to lack of employment opportunities and better salary or remuneration. For instance; an employee become continuous committed when an employee chooses to stay because remuneration and salary will not go any better if they quit to another employer. Continuous commitment Questionnaire had six (6) items. Example items in the questionnaire included..."It would be very hard to leave the organization right now even if I wanted to"...."Too much of life would be interrupted if I decided to leave the organization"....."Right now staying in the organization is as much of necessity as of desire".... Perception of continuous commitment was measured in five scale represented by 1= Strongly Disagree; 2= Disagree; $3=$ Undecided; 4= Agree and 5= Strongly Agree

\section{Demographic questionnaire scale}

Demographic data were collected using Social Demographic Data Questionnaire designed and developed by the researcher pending customized study needs. It is composed of 4 items namely sex, age, working experience and organization type. Respondents were restricted to describe their characteristics in predetermined categories of responses. For example; age was categorized in three classes namely [a] Less than 35 [b] Between 35 and 40 [c] Greater than 40. Likewise, experience was categorized in two classes namely [a] Less than 10 years [b] Greater than 10 years. All respondents needed were to put a tick in respective box.

\subsubsection{Data analysis}

Principal Component Analysis (PCA): In study 1; collected data were analyzed to assess if they were component of common constructs in questionnaire under independence scrutiny. Analysis was possible through Principal Component Analysis (PCA). PCA is defined as a multivariate method that analyses data such that; observations are explained by various inter-correlated quantitative dependent variables (Abdi \& Williams, 2010). It focuses in taping essential data information to embody data as a set of new orthogonal variables called principal components. It does so by showing data pattern of observation similarity and variables as points in maps (Abdi \& Williams, 2010). Accordingly, data collected in the first study got screened to establish scale constructs that were component of common constructs. In other words, researcher screened constructs purported to make three sub-scales of Allen and Meyer's Organizational Commitment Scale namely affective, normative and continuous Organizational Commitment. In SPSS, Principal Component Analysis was employed such that all items (in individual scale) be forced extracted in respective to see loading extent that decides fates (of items) in the scale. Constructs retaining criteria was factor loadings of .05 or better off. Therefore, all constructs that recorded at least 0.50 factor loading were to be retained for further analysis in the second study.

\subsubsection{Ethical Considerations}

Compliance with ethical research requirement was not optional; hence it was not compromised either. Thus far, as this study is concerned, deliberate efforts were devoted to comply with ethical requirements in academic research. Some of them included: - First: Asking formal permission to conduct a study in participating organizations. Permission quest was accompanied by conversation and production of data collection instruments to give confidence on the extent of harmless the study was. Second: the researcher agreed fully with the anonymity requirements of the participating councils; where they requested names to not appear in any report of the paper. That is why names like DC-PS, DC-MS, and MC-MS appears in place of participating organizations. DC-FS stands for District Council in First Study; DC-MS stand for District Council in Main Study and MC-MS stand for Municipal Council for Main Study. Thirdly: Individual privacy and dignity was also adhered in this study. Using internal research assistant employees were informed that participation was voluntary and anonymity was kept in a stringent manner. Fourth: collected data were kept in special envelop and kept in researcher's locker to ensure nobody could access the same. This was important as security of data collected was emphasized by all council administration before permission was granted. 


\subsection{Study 2}

General objective of the study 2 was to analyze reliability and validity of the data collected by Allen and Meyer's Organizational Commitment questionnaire. Specific objectives were three-fold: - One: Test data normality as an important task before engaging vigorous statistical techniques. Two: Establish Allen and Meyer's Organizational Commitment questionnaire internal consistency and see whether or not; it collects what it ought to collect in Tanzanian data. Three: Establish whether or not; Allen and Meyer's Organizational Commitment questionnaire exhibit sound validity, in Tanzania data.

\subsubsection{Sample}

Available officers were counted to be 277 (District Council in study 2) and 393 (Municipal Council in Study 2) making a total of 670 available respondents. Sample in District Council had 42 supervisors and 235 nonsupervisors. Conversely, in Municipal Council; the sample included 52 supervisors and 341 non-supervisors. The study employed Yamane (1967) formula to determine the actual sample size of the study. The basis for selecting this formula includes its simplicity, incorporation of precision level and confidence level in a given population. In mathematical terms the formula (District and Municipal) can be depicted as: -

Sample Size: District Council Study 2

$\boldsymbol{n}=\frac{277}{1+277\left(0.05^{2}\right)} \quad=163$

Sample Size: Municipal Council Study 2

$n=\frac{393}{1+393\left(0.05^{2}\right)} \quad=198$

\subsubsection{Data analysis}

The same questionnaires used in study 1; were also used in study 2. However, in study 2; two organizations were involved and more analyses were engaged. In fact, three analyses were conducted including Normality Analysis; Reliability Analysis and Confirmatory Factor Analysis (CFA).

\section{Normality Analysis}

Statistical technique employed in this study presumes the distribution of scores on the outcome variable is substantively normal. Apparently, when data are not normally distributed, related normal and chi-square observations are rendered inaccurate and thus $\mathrm{T}$ and $\mathrm{F}$ tests lack command in validity scrutiny (Judge et al. 1985). Consequently, it was fundamental to explore data normality using graph in Allen and Meyer's Organizational Commitment Scale. Indeed; this was done before any statistical analysis was done. Certainly; several normality analyses exist and each is got some merits and demerits (Shukla, 2015). This study, however, tests data normality using non-significance results, skewness and kurtosis values. This method is best when data set is large enough $(100+)$; otherwise, it will give just rough idea about symmetry of the distribution (D'Agostino, 1990). Data to this study is quite large enough; thus, safe to employ Skewness and Kurtosis tests in data normality. Data analysis and respective Interpretation based on D'Agostino, (1990) rule of thumb; that skewness less than -1 or greater than +1 ; the distribution is highly skewed. Skewness between -1 and -.5 or between +.5 and +1 ; the distribution is moderately skewed. Then again, skewness between -.5 and +.5 , the distribution is approximately symmetric and therefore there is no need of employing transforming techniques (Shukla, 2015). Distribution of scores in the scale was then subjected to normality test.

\section{Reliability Analysis}

Questionnaire or Scale Reliability refers the degree to which it consistently measures the construct it is purported to measure (Field, 2015). According to Field; scale reliability is to the effect that; if questionnaire is completed in different points; researchers should receive relatively the same scores. Cronbach's alpha is one of the popular methods of testing questionnaire reliability (Cronbach, 1951). Study suggests; Cronbach's alpha that ranges between 0.7 to 0.8 is good internal consistency; as it indicates questionnaire is reliable (Kline, 1999). Similar studies have reported reliability of Allen and Meyer's Organizational Commitment Scale; at the tune of 0.91 (Hannang, 2005). Data analysis and interpretation in this study; assessed questionnaire reliability using Cronbach's alpha 0.70 and Corrected Item-Total Correlation better than 0.30 . These values would not only indicate good internal consistency; but also, the questionnaire is not measuring something else (Cortina, 1993)

\section{Confirmatory Factors Analysis}

Confirmatory factor analysis (CFA) is a statistical technique deployed to confirm the factor structure of a set of observed variables (Child, 1990). Child suggests; CFA lets researchers test hypothesis that "there is significant relationship between observed variables and respective latent constructs". Researchers utilize confirmatory factor analysis (CFA) for construct validation in questionnaires (Prudon, 2015)). In particular, when deployed in multidimensional analyses; covariance matrix is computed and compared to scores of subjects. It follows that CFA is applied to confirm whether or not; purported factor is not contradicted by predetermined matrix (Prudon, 2015). Thus; CFA is considered a very sophisticated statistical technique; that is conducted using Structural Equation Modeling (SEM) in Amos software. Once three models (Affective, Normative and Continuous Commitment) were specified; AMOS Software was commanded to replicate two models (males and females' models) for comparison purposes. Much as sample size was large enough $(200+)$; it was possible to utilize other indices such as RMR 
(Root Mean Square Residual), GFI (Goodness Fit Index), TLI (Tucker-Lewis Index), CFI (Comparative Fit Index) and RMSEA (Root Mean Square Error of Approximation). Chi-square is represented by CMIN value in the AMOS output which put into overt the differences in the model of interest. It is accompanied by p-value that if greater than .05 suggest model fit collected data.

\section{Results and Discussion}

\subsection{Study Respondents}

Allen and Meyer's Questionnaire (1990); was utilized to collect data in Study 1 to be sure; three scales actual are separate. It was meant to assess whether or not; Allen and Meyer's Questionnaire (1990) forms component of common constructs using Tanzanian data. A total of 104 respondents from first District Council (DC) completed and returned questionnaires. Respondents were 56 (53.8\%) females and 48 (46.2\%) males. On age footings; 52 $(50 \%)$ respondents of age below 35 completed questionnaires. Further, respondents with age between 35-40 $(38.5 \%)$ and those above $40(11.5 \%)$ returned completed questionnaires. Respondents with below $10(61.5 \%)$ years of experience dominated respondents; followed by counterpart with age more than $10(38.5 \%)$ at work. In study 2; a total of 214 respondents completed questionnaires. Out of 214; females were 177 (64.6\%) and males were 97 $(35.4 \%)$. Questionnaires were handed to potential respondents on convenient approach by prepared data collectors. On age terms; younger generation lead response at the tune of 92 (43\%); between 35-40 made $79(36.9 \%)$; followed by $43(20.1 \%)$ older generation. Equally, 131 (61.2\%) respondents of lesser experienced at work took lead as well in response. Respondents with experience more than 10 made $83(38.8 \%)$. At a glance the study attracted a total of 318 involved in study 1 and 2 . Table 1 summarizes respondent with selected characteristics.

Table 1: Demographic Characteristics

\begin{tabular}{|c|c|c|c|c|}
\hline \multirow[t]{2}{*}{ Characteristics } & \multicolumn{2}{|c|}{ Respondents } & \multicolumn{2}{|c|}{ Percentage } \\
\hline & Study-1 & Study-2 & Study-1 & Study-2 \\
\hline \multicolumn{5}{|l|}{ Sex } \\
\hline $\mathrm{F}$ & 56 & 177 & $53.8 \%$ & $64.6 \%$ \\
\hline $\mathrm{M}$ & 48 & 97 & $46.2 \%$ & $35.4 \%$ \\
\hline \multicolumn{5}{|l|}{ Age } \\
\hline$\geq 35$ & 52 & 92 & $50 \%$ & $43 \%$ \\
\hline $35-40$ & 40 & 79 & $38.5 \%$ & $36.9 \%$ \\
\hline$\leq 40$ & 12 & 43 & $11.5 \%$ & $20.1 \%$ \\
\hline \multicolumn{5}{|l|}{ Council } \\
\hline DC-1 & 104 & & $53.6 \%$ & \\
\hline DC-2 & & 112 & & $52.3 \%$ \\
\hline $\mathrm{MC}$ & & 102 & & $47.7 \%$ \\
\hline \multicolumn{5}{|l|}{ Experience } \\
\hline$\geq 10$ & 64 & 131 & $61.5 \%$ & $61.2 \%$ \\
\hline$\leq 10$ & 40 & 83 & $38.5 \%$ & $38.8 \%$ \\
\hline
\end{tabular}

$\mathrm{DC}=$ District Council; $\mathrm{MC}=$ Municipal Council

\subsection{Principal Component Analysis}

Principal Component Analysis (PCA) was employed to establish scale items that were component of common constructs. Three separate Principal Component Analyses was deployed to uncover the structure of the respective scales. Analysis (PCA) on the affirmative (six items), normative (six items) and continuous (six items) commitment to determine degree to which they load on common component was conducted using data collected (Study 1) in Tanzanian culture. Given the solid theoretical background of the scale; the researcher decided to analyze "factor extraction" using fixed number of factor and so specifying number to extract (also known as forced factor extraction)

\section{Affective Commitment}

Affective Commitment Scale (ACS) was subjected to Principal Component Analysis (PCA). Results (affective commitment) on Correlation matrix reported good correlations commendable for analysis and go-ahead interpretation. Table 2 (Affirmative Commitment) shows the model was significant indicated by $\mathrm{p}<.05$; and $\mathrm{KMO}$ greater than 0.6. Check out of factor loading in the last column of the table 2; suggest no coefficient or loading variable was worse than 0.9 . Hence all 6 variables seem to load on common component; in this case Affective Commitment Scale. Then, it is plausible to utilize these variables for the other analyses in this study (Study 2) Results are summarized in table 2 
Table 2: Factor loading for Affective Commitment Scale (ACS)

\begin{tabular}{|c|c|c|c|c|c|c|c|}
\hline \multirow[t]{2}{*}{ Variable } & \multirow[t]{2}{*}{ Total items } & \multirow[t]{2}{*}{ Deducted items } & \multirow{2}{*}{$\begin{array}{l}\text { Retained } \\
\text { items }\end{array}$} & \multirow[t]{2}{*}{ KMO } & \multicolumn{3}{|c|}{ Bartlett's Test of Sphericity } \\
\hline & & & & & $\chi 2$ & df & Sig. \\
\hline \multirow[t]{2}{*}{ ACS } & 6 & 0 & 6 & .723 & 93.135 & 15 & $.000^{*}$ \\
\hline & & & & & & & $\begin{array}{l}\text { Factor } \\
\text { loading }\end{array}$ \\
\hline \multicolumn{7}{|c|}{ 1. I would be happy to spend the rest of my career with the organization } & .90 \\
\hline \multicolumn{7}{|c|}{ 2. I really feel as if the organization's problems are my own } & .94 \\
\hline \multicolumn{7}{|c|}{ 3. I do not really feel part of a family at my organization (R) } & .97 \\
\hline \multicolumn{7}{|c|}{ 4. I do not feel emotionally attached to the organization (R) } & .97 \\
\hline \multicolumn{7}{|c|}{ 5. The organization has a great deal of personal meaning to me } & .96 \\
\hline \multicolumn{7}{|c|}{ 6. I do not feel a sense of belonging to the organization (R) } & .98 \\
\hline
\end{tabular}

$\mathrm{KMO}=$ Kaiser-Meyer-Olkin Measure of Sampling Adequacy

Bartlett's Test of Sphericity measure of model significance

$\chi 2=$ Approx. Chi-Square

Hitherto, Results for Normative Commitment Scale (NCS) indicated the entire analysis had no less than 0.3 correlation problems in most elements and so safe to go ahead with screening. Table 3 showed the model was significant as $\mathrm{p}<.05$; and $\mathrm{KMO}$ greater than 0.6 . Also, scanning through factor loading column, no single variable scored loading less than 0.5 ; the minimum; indicating all variables loads to common component. On this background all six items (that form Normative Commitment Scale) are proposed for other analysis (Study 2). Results are summarized in table 3

Table 3: Factor loading for Normative Commitment Scale (NCS)

\begin{tabular}{|l|l|l|l|l|l|l|l|}
\hline Variable & $\begin{array}{l}\text { Total } \\
\text { items }\end{array}$ & $\begin{array}{l}\text { Deducted } \\
\text { items }\end{array}$ & $\begin{array}{l}\text { Retained } \\
\text { items }\end{array}$ & \multicolumn{2}{l|}{ KMO } & \multicolumn{2}{l|}{ Bartlett's Test of Sphericity } \\
\hline & & & & & $\chi \mathbf{2}$ & df & Sig. \\
\hline NCS & 6 & 0 & 6 & .819 & 327.143 & 15 & $.000^{*}$ \\
\hline & & & & & $\begin{array}{l}\text { Factor } \\
\text { loading }\end{array}$ \\
\hline
\end{tabular}

$\mathrm{KMO}=$ Kaiser-Meyer-Olkin Measure of Sampling Adequacy

Bartlett's Test of Sphericity measure of model significance

$\chi 2=$ Approx. Chi-Square

Further, Results for Continuous Commitment Scale (CCS) on Correlation matrix was far from 0.3 cutoff point minimum as most correlations were greater than rule of thumb point. As it can be seen in the table 4 results documented $\mathrm{p}<.05$; and KMO greater than 0.6 which indicated significant model was achieved. Looking at factor loading column the lowest loading was 0.81 which proposed all six variables had strong association with Continuous Commitment Scale. It is proposed, subsequent analysis should use these six variables in Continuous Commitment Scale. Results are summarized in table 4 
Table 4: Factor loading for Continuous Commitment Scale (CCS)

\begin{tabular}{|c|c|c|c|c|c|c|c|}
\hline \multirow[t]{2}{*}{ Variable } & \multirow[t]{2}{*}{ Total items } & \multirow[t]{2}{*}{ Deducted items } & \multirow{2}{*}{$\begin{array}{l}\text { Retained } \\
\text { items }\end{array}$} & \multirow[t]{2}{*}{ KMO } & \multicolumn{3}{|c|}{ Bartlett's Test of Sphericity } \\
\hline & & & & & $\chi^{2}$ & df & Sig. \\
\hline $\mathbf{C C S}$ & 6 & 0 & 6 & .897 & 368.197 & 15 & $.000^{*}$ \\
\hline & & & & & & & $\begin{array}{l}\text { Factor } \\
\text { loading }\end{array}$ \\
\hline \multicolumn{7}{|c|}{ 1. It would be very hard to leave the organization right now even if I wanted to. } & .81 \\
\hline \multicolumn{7}{|c|}{ 2. Too much of life would be interrupted if I decided to leave the organization } & .87 \\
\hline \multicolumn{7}{|c|}{ 3. Right now staying in the organization is as much of necessity as of desire } & .88 \\
\hline \multicolumn{7}{|c|}{ 4. I believe I have too few options to consider leaving the organization now } & .91 \\
\hline \multicolumn{7}{|c|}{ 5. One of the negatives of leaving the organization would be scarcity of alternatives } & .88 \\
\hline \multicolumn{7}{|c|}{ 6. Leaving would require considerable sacrifice and other organizations benefits do no } & .68 \\
\hline
\end{tabular}

$\mathrm{KMO}=$ Kaiser-Meyer-Olkin Measure of Sampling Adequacy

Bartlett's Test of Sphericity measure of model significance

$\chi 2=$ Approx. Chi-Square

\subsection{Normality Analysis}

Organizational Commitment Questionnaire was tested against normality using Kolmogorov-Smirnov Statistics, Skewness and Kurtosis values. Affective, Normative and Continuous Commitment was run together for normality assessment. Results produced significant normality test at $\mathrm{p}<.05$; suggesting distribution scores should be skewed somewhere in the distribution graph which possibly violate normality assumption. As for skewness, scores in the scale was relatively clustered to the left of the distribution graph with low values (Skewness .265) while kurtosis indicates score distribution roughly flat with good number of cases in the extremes (Kurtosis -1.427). Supports to this observation may be seen in Total Organizational Commitment distribution graph below with relatively flat bell shape pushed to the right-hand side of the graph. As long as skewness value range between -.5 and +.5 (approximately normal according to Bulmer); no extra transformation of data was attempted. See Graph 1



Graph 1: Organizational Commitment Normality Graph

\subsection{Reliability Analysis}

Using data collected in the Study 2; reliability of all scales was examined using Cronbach alpha analyses. Results indicated combined Organizational Commitment Scale scored 0.91 Cronbach alpha. An assessment of Corrected Item-Total Correlation indicated all values in the column were better than .30. That meant, the degree to which each item in the scale correlates with the total score was normal and therefore it was not measuring something different from the scale as a whole. Further, Cronbach alpha 0.91 suggest the extent to which Organizational Commitment Scale is consistent when applied to Tanzanian data. That is to say; if it is used several times (In Tanzanian context; it is likely to produce the same results. Previous studies; for example, Allen \& Meyer (1990); registered sound Organizational Commitment Scale internal consistency to the tune of Cronbach alpha coefficient 
of 0.87 (Affective Commitment), 0.75 (Continuance Commitment), 0.79 (Normative Commitment) and 0.80 for Organizational Commitment Questionnaire in general. Consequently, this study, confirms previous study findings that Organizational Commitment Questionnaire is reliable in data collection; even when used in non-western cultures. It sits proper, therefore, to suggest Organizational Commitment Scale (Affective, Continuous and Normative Commitment) is reliable measure of Organizational Commitment in Tanzanian data.

\subsection{Confirmatory Factors Analysis}

Data collected in Study 2 were used to establish validity of the Allen and Meyer's Organizational Commitment Scale in Tanzanian culture. Once Organizational Commitment model were specified; AMOS was commanded to replicate two models (males and females models) for comparison purposes. The sample size (200+) allowed analyses of other fit indices namely RMR (Root Mean Square Residual), GFI (Goodness Fit Index), TLI (TuckerLewis Index), CFI (Comparative Fit Index) and RMSEA (Root Mean Square Error of Approximation). Chi-square is represented by CMIN value in the AMOS output which put into overt the differences in the model of interest. It is accompanied by $\mathrm{p}$-value that if greater than .05 suggested model fits collected data. Table 5 represents Chisquare (CMIN) of the Organizational Commitment Scale.

Table 5: Comparison of models based on Chi-square

\begin{tabular}{|c|c|c|c|c|c|}
\hline \multicolumn{6}{|c|}{ Organizational Commitment Scale } \\
\hline Models & NPAR & CMIN & DF & $\mathbf{P}$ & CMIN/DF \\
\hline Final model & 72 & 616.232 & 270 & .000 & 2.282 \\
\hline Saturated model & 342 & .000 & 0 & & \\
\hline Independence model & 36 & 2619.128 & 306 & .000 & 8.559 \\
\hline
\end{tabular}

Table 5; shows summary of chi-square results in the quest to determine if path coefficients for males and females are equal and so determine whether designed models fit data. On this ground, it will also be possible to determine validity of scales and data used in this study. Using SEM specified models were assessed if males and females respondents had path coefficients that are invariant ( $\mathrm{p}$-greater than .05). Table 5 is interpreted by scanning down to column three (CMIN which stand for chi-square); Column four (DF ie Degree of Freedom) and Column five ( $\mathrm{P}$ also known as probability value). For model fit $\mathrm{p}$-value needed to be greater than .05 showing no difference of path coefficient between male and female respondents. However, as expected, Organizational Commitment model had p-value lesser than .05; hence suggesting males and females are not equal as path coefficients are variant (bad validity). So far, it is well known that Chi-square has issues on certainty/reliability and it is no wonder other three models fitted badly by having significant results instead of insignificant that would fit models relatively better ( $p$-value greater than .05). Other indices fit is checked in table 6

Table 6: Comparison of models based on Root Mean Square Residual (RMR) and Goodness Fit Index (GFI)

\begin{tabular}{|l|r|r|r|r|}
\hline Models & & & & \\
\hline Performance Appraisal Quality Scale & RMR & GFI & AGFI & PGFI \\
\hline Final model & .092 & .832 & .660 & .578 \\
\hline Saturated model & .000 & 1.000 & & \\
\hline Independence model & .597 & .209 & .116 & .187 \\
\hline
\end{tabular}

Following poor reliability and validity of chi-square particularly in large sample like of this study; RMR and GFI indices fit was opted to test if path coefficients for males and females are equal. Scanning down to the first column labeled RMR in table 6; all values for Organizational Commitment scale tested for equality are well close to zero. That is; Organizational Commitment documented .092. Results of this nature speak loudly that the specified model fit collected data well and so validity of the scale to Tanzanian context. In the same vein, perusing down in the column labeled GFI table 7; Organizational Commitment documented .832. This is closer to .90 as recommended. Making reference to Organizational Commitment which registered .832; it can be argued that specified model and model correspond better by $82.3 \%$ than independence model calculated using the very same data. Under GFI, it is ruled out that there is a tally between specified model and data as males and females are invariant in path coefficients.

Table 7: Comparison of models based on Comparative Fit Index (CFI) and Tucker-Lewis Index (TLI)

\begin{tabular}{|l|r|r|r|r|r|}
\hline Organizational Commitment Scale & & & & & \\
\hline Models & NFI & RFI & IFI & TLI & CFI \\
\hline Final model & .765 & .733 & .853 & .900 & .901 \\
\hline Saturated model & 1.000 & & 1.000 & & 1.000 \\
\hline Independence model & .000 & .000 & .000 & .000 & .000 \\
\hline
\end{tabular}

To establish that male and females match the assumption that they are invariant; specified models were compared using Comparative Fit Index (CFI) and Tucker-Lewis Index (TLI). Values closer to .90 or above suggested model-data fit because it is the sign that males and females are equal in path coefficient scores; hence validity of the used scale. Interpretation dwells in column labeled TLI and CFI. Apparently, skimming down (in 
TLI) across the scale listed on the extreme left no value is worse than .90 which is soundly equal to .90 as recommended; this posit the fit on the final model was $90 \%$ far better than independence model calculated from the same data (Organizational Commitment). It sits proper to suggest not only the data fits the model but also validity of scale is good enough using TLI as comparison criteria. Contrary, values of CFI across the scale in table 6 display no value below .901; which is safe to rule out that there is sufficient evidence on the model-data correspondence and so does scale validity as male and female displays no differences. Stated otherwise there are invariant in path coefficient scores when CFI is taken into considerations. Table 7 compares RMSEA

Table 7: Comparison of models based on Root Mean Square Error of Approximation (RMSEA)

\begin{tabular}{|l|r|r|r|r|}
\hline Performance Appraisal Quality Scale & \multicolumn{5}{|l|}{ PCLOSE } \\
\hline Models & RMSEA & LO 90 & HI 90 & PCL \\
\hline Final model & .058 & .040 & .086 & .000 \\
\hline Independence model & .189 & .182 & .196 & .000 \\
\hline
\end{tabular}

Root Mean Square Error of Approximation (RMSEA) is pointed out to be one of the valuable fit indices to replace unreliable chi-square when sample size grows larger. For the model fit, values in column labeled RMSEA should not be larger than .06 rule of thumb fit. Looking through down this column (in final model); Organizational Commitment Scale had values just below cutoff value .06 indicating model fit

\section{Conclusions and Recommendations}

\subsection{Conclusions}

Allen and Meyer's Organizational Commitment Questionnaire (1990) were assessed for Constructs independence, Normality, Reliability and Validity using Tanzanian data. In so doing; Principal Component Analysis, Normality Analysis, Reliability Analysis and Confirmatory Factor Analysis were utilized. Primarily, the objective of the study was twofold: -

1. To examine whether or not; Allen and Meyer's Questionnaire (1990) namely Affective, Normative and Continuous Commitment questionnaires are independent constructs using Tanzanian data

2. To investigate the validity, reliability and normality levels of Allen and Meyer's Questionnaire (1990) using Tanzanian data

The study was gained significance on account of three major reasons: - One: Allen and Meyer's Questionnaire (1990) was developed and validated in western cultures. Two: Reviewed Psychometric Properties Findings on Allen and Meyer's Questionnaire (1990) shown inconsistent and contradictory results. Three: there is general lack of validity, reliability, normality and construct independence in Tanzanian culture. Consequently; the following are conclusions drawn by this study: -

- Collected data using Allen and Meyer's Questionnaire (1990) Affective, Normative and Continuous Questionnaires; was subjected to forced factor extraction. The three questionnaires produced sound correlations commendable for use in other analysis. They further; produced significant models $(\mathrm{p}<.05$; and KMO greater than 0.6.). No coefficient or loading variable was worse than 0.9. All eighteen (18); loaded on common components (affective, normative and continuous); which suggested no need for scale adjustments. It is therefore safe to utilize these scales (affective, normative and continuous) for collecting Organizational Commitment responses (in Tanzanian context) as they carry independent constructs

- Allen and Meyer's Questionnaire (1990) Affective, Normative and Continuous Questionnaires data was scrutinized using Kolmogorov-Smirnov Statistics, Skewness and Kurtosis minimum acceptable values. Cumulative scale (Affective, Normative and Continuous Commitment) produced significant normality test at $\mathrm{p}<.05$. Skewness, scores in the scale was relatively clustered to the left of the distribution graph with low values (Skewness .265) while kurtosis indicated score distribution roughly flat with good number of cases in the extremes (Kurtosis -1.427). Skewness value ranged between -.5 and +.5 (approximately normal according to Bulmer); no extra transformation of data was required. It is therefore concluded that Allen and Meyer's Questionnaire (1990) Affective, Normative and Continuous Questionnaires data produced normal distribution characteristics in Tanzanian context. This permitted further statistical analysis in Tanzanian data.

- Data collected by Allen and Meyer's Questionnaire (1990); was subjected to vigorous Cronbach Alpha Analysis. It registered sound Organizational Commitment Scale internal consistency to the tune of Cronbach alpha coefficient of 0.87 (Affective Commitment), 0.75 (Continuance Commitment), 0.79 (Normative Commitment) and 0.80 for Organizational Commitment Questionnaire in general. It was deduced; Scales internal consistency was adequately good. On this basis; it is concluded; Allen and Meyer's Questionnaire (1990); is reliable in data collection; even when used in non-western cultures.

- Data collected by Allen and Meyer's Questionnaire (1990); was tested its validity using Confirmatory Factors Analysis (CFA) in Amos software. Fit indices namely RMR (Root Mean Square Residual), GFI (Goodness Fit Index), TLI (Tucker-Lewis Index), CFI (Comparative Fit Index) and RMSEA (Root Mean 
Square Error of Approximation) suggested models fit with Tanzanian data. It is concluded that; Allen and Meyer's Questionnaire (1990); produced sound validity properties using Tanzania data. Tanzanian researchers may safely use the questionnaire to collect data pertaining to Organization Commitment studies.

\subsection{Recommendations}

Reviewed literature in this study consistently indicated; questionnaire response bias; caused by whatever sources; is substantial problem in studies. A major problem noteworthy mentioning is that; it impacts statistical analyses and yield distorted results and inaccurate estimates (Swaidan, 2018). According to Swaidan there are various techniques to adjust the impact of response bias; but all of them clustered under during data collection and during data analyses. This study proposes three techniques that can be utilized to reduce or eliminate response bias during data analyses. These are

\section{- Social Desirability Detection Scale Technique}

By definition; Social desirability refers respondents' propensity to rate questionnaire items such that respondents appear good; rather than actual honest rating (Holtgraves, 2004). The behaviour is significant in range of questionnaire responses across cultures and influences response bias (Fleming, 2012). As technique; Social Desirability Detection Scale Technique; works best during data collection phase (Lambert et al, 2016). Social Desirability Detection Scale can be used to detect social desirability by employing statistical analyses where data adjustment may be possible prior main analyses. For example; Preiss et al, (2015) tested hypothesis that; in comparison to controls; respondents from legal cadre shows similar behavior towards social desirability. Findings suggested statistically significant differences between legal cadre group and control group. In conclusion; researchers may opt to detect response bias and adjust the same before final analyses.

\section{- Score Standardization Technique}

Response bias has also been adjusted using Score Standardization Technique. Often it is best when extreme responding is an issue to data collection (Brinker, 2002) in one hand. On the other; it is also useful when there is response bias associated by variation in cultural background. Score Standardization Technique can be utilized in two ways namely Z-scores and Deviation scores. On the former (Z-scores); Respondents raw scores are transformed across variables to z-scores by standardizing the distribution of their responses (Brinker, 2002). On the latter (Deviation scores); Respondents raw scores are transformed using deviation scores drawn via ipsatization (Fischer, 2004)

\section{- $\quad$ Response Bias Predictors Techniques}

This technique is to the effect that response bias must not only get identified; but also quantified; so that to pave the way for bias reduction or elimination (Swaidan, 2018). According to Swaidan; once response bias is detected and quantified; it can be adjusted statistically and thus improve collected data accuracy and statistical estimates. In practice researchers simply detect predictors of response bias in questionnaires and measure the same. It follows that identified response bias predictors are statistically controlled for; during actual analyses.

\section{References}

Abdi \& Williams, (2010): Principal component analysis; WIREs Computational Statistics; Vol, 2, pp 433 - 459

Allen, N. and Meyer, J.P. (1990), "The measurement and antecedents of affective, continuance and normative commitment to the organization", Journal of Occupational Psychology, Vol. 63, pp. 1-18.

Abbas Qasir, \& Khanam Sarwat Jahan (2013); Psychometric properties of urdu translation and Adaptation of organizational commitment Questionnaire (OCQ) in Pakistan; Asian Journal of Management Sciences \& Education; Vol.2 No 4. Pp, $240-248$

Addae Helena, Praveen Parboteeah; \& Nickler Velinor (2008); Role stressors and organizational commitment: public sector employment in St Lucia; International Journal of Manpower; Vol. 29 No. 6, pp. 567-582

Allen, N. J. \& Meyer, J. P., (1997) Commitment in the workplace: Theory, research, and application. Thousand Oaks, CA: Sage Publications.

Anna, D (2017); Article on classification of response scale characteristicsthat affect data quality: a literature review; Found at https://doi.org/10.1007/s11135-017-0533-4 on 12/10/2020

Billiet, J. B., \& Davidov, E. (2008). Testing the stability of an acquiescence style factor behind two interrelated substantive variables in a panel design. Sociological Methods \& Research, 36, 542-562.

Beanland, C., Schneider, Z., LoBiondo-Wood, G. and Haber, J. (1999). Nursing research: methods, critical appraisal and utilisation. Harcourt: Sydney, Australia.

Bogner, K., \& Landrock, U. (2016). Response Biases in Standardised Surveys. GESIS Survey Guidelines. Mannheim, Germany: GESIS - Leibniz Institute for the Social Sciences. doi: 10.15465/gesis-sg en 016

Brinker, G. D. (2002). Using standard scores to control for extreme response style bias. Journal of Applied 
Sociology,

$19(2)$

81-99.

Retrieved

from http://www.jstor.org/stable/43481457?seq=1\#page_scan tab_contentsCab

Buchanan, B. ( 1974). Building organizational commitment: The socialization of managers in work organizations. Administrative Science Quarterly, 19, 533-546.

Cook, J., \& Wall, T. (1980). New work attitude measures of trust, organizational commitment and personal need nonfulfilment. Journal of Occupational Psychology, 53(1), 39-52. doi: 10.1111/j.2044-8325.1980.tb00005.x

Chen, Z.X. and Fransesco, A.M. (2003), "The relationship between the three components of employee commitment and employee performance in China", Journal of Vocational

Child, D. (1990). The essentials of factor analysis, second edition. London: Cassel Educational Limited.

Dunham et al (1994); Organizational Commitment: The utility of an integrative definition. Journal of Applied Psychology. Vol. 79, pp, 370-380

Falkenburg B. \& Schyns B. (2007); Work satisfaction, organizational commitment and withdrawal behaviours; Management Research News, Vol. 30 No. 10, pp. 708-723

Fischer, R. (2004). Standardization to account for cross-cultural response bias: A classification of score adjustment procedures and review of research in JCCP. Journal of CrossCultural Psychology, 35, 263-282

Fleming, P. (2012). Social desirability, not what it seems: a review of the implications for self-reports. Int. J. Educ. Psychol. Assess. 11, 3-22.

Goulet, L.R. and Frank, M.L. (2002), "Organizational commitment across sectors: public, non-profit, and for profit”, Public Personnel Management, Vol. 31, pp. 201-10.

Harumi Cheryl A. (2011); Cross-Cultural Difference in Response Styles; PhD Thesis; Washington State University

Holtgraves, T. (2004). Social desirability and self-reports: testing models of socially desirable responding. Pers. Soc. Psychol. Bull. 30, 161-172. doi: 10.1177/0146167203259930

Jaros, S. (2007). Meyer and Allen model of organisational commitment: Measurement issues. The Icfai Journal of Organisational Behaviour, 4(4), 7-25.

Jaros, S.T., Jermier, J. M., Koehler, J.W., \& Sincich, T. (1993). Effects of continuance , affective an moral commitment on the withdrawal process: an evaluation of eight structural equation models, Academy of Management Journal, 36,951-995.

Karim Noor Harun Abdul \& Noor Noor Hasrul Nizan Mohammd (2006); Evaluating the psychometric properties of Allen and meyer's organizational commitment scale: A cross cultural application among Malaysian academic librarians; Malaysian Journal of Library \& Information Science, Vol.11, no.1, July 2006: 89-101

King, M. and Bruner, G. (2000). Social desirability bias: a neglected aspect of validity testing. Psychology and Marketing, 17(2):79-103

Kanning P. \& Hill. A (2013); Validation of the Organizational Commitment Questionnaire (OCQ) in six Languages; Journal of Business and Media Psychology, Vol 4, Issue 2, 11-20,

Krosnick, J. A. (1999a). Maximizing questionnaire quality. In J. P. Robinson, P. R. Shaver, \& L. S. Wrightsman (Eds.), Measures of Political Attitudes (pp. 37-57). San Diego: Academic Press

Kulas, J.T., Stachowski, A.A.: (2009); Middle category endorsement in odd-numbered Likert response scales: associated item characteristics, cognitive demands, and preferred meanings. J. Res. Pers. 43, 489-493 doi:10.1016/j.jrp.2008.12.005

Lambert, C. E., Arbuckle, S. A., \& Holden, R. R. (2016). The Marlowe-Crowne Social Desirability Scale outperforms BIDR Impression Management Scale for identifying fakers. The Journal of Research in Personality, 61, 80-86. doi:10.1016/j.jrp.2016.02.004

McCaul, H.S., Hinsz, V.B. and McCaul, K.D. (1995), “Assessing organizational commitment: an employee’s global attitude toward the organization", Applied Behavioral Science, Vol. 1, pp. 80-90.

Mathieu, J.E. \& Zajac, D.M. (1990), “A review and meta-analysis of the antecedents, correlates and consequences of organizational commitment', Psychological Bulletin, Vol. 2, pp. 171-94.

Mawell G. \& Steele G. (2003). Organizational Commitment: A study of managers in hotels; International Journal of Contemporary Hospitality Management; V 15 (7); P 362 - 369

Maertz, C.P., Griffeth, R.W., Campbell, N.S. and Allen, D.G. (2007), "The effects of perceived organizational support and perceived supervisor support on employee turnover", Journal of Organizational Behavior, Vol. 28, pp. 1059-75.

Maqsood et al, (2012); Validation of the Three-Component Model of Organizational Commitment Questionnaire; FWU Journal of Social Sciences, Vol. 6, No. 2, pp, 135-145

Meyer, J.P., Herscovitch, L. (2001). Commitment in the workplace: Toward a general model, Human Resource Management Review, 11, 299-326

Meyer, J. P., \& Allen, N. J. (2004). TCM employee commitment survey: Academic users' guide. West Ontario, Canada: University of Western Ontario.

Meyer, J. P. \& Allen, N. J. (1997). Commitment in the Workplace. Theory, Research, and Application. Thousand Oaks, CA: Sage. 
Mowday, R. T., Steers, R. M., \& Porter, L. W. (1979). The measurement of organisational commitment. Journal of Vocational Behaviour, 14, 224-247.

Mortel T . F (2015); Faking it: social desirability response bias in self-report research; Australian Journal of Advanced Nursing Volume 25 Number 4

Nederhof, A. (1985). Methods of coping with social desirability bias: a review. European Journal of Social Psychology, 15(3):263-280

Norma Betanzos-Díaz, Cyntia Shugey Rodríguez-Loredo1 and Francisco Paz-Rodríguez (2007); Development and validation of a questionnaire on normative organizational commitment: A pilot study in Mexicans workers; Anales de Psicología, vol. 33, núm. 2, mayo, pp. 393-402

Pais et al, (2014); Affective and continuance commitment in call centres: validation of Meyer and Allen questionnaire; SGEM International Multidisciplinary Scientific Conference son Social Sciences and Arts

Preiss M, Mejzlíková T, Rudá A, Krámský D and Pitáková J (2015) Testing the Level of Social Desirability During Job Interview on White-Collar Profession. Front. Psychol. 6:1886. doi: 10.3389/fpsyg.2015.01886

Prudon, P. (2015) Confirmatory factor analysis as a tool in research using questionnaires: a critique. Comprehensive Psychology, 4, 10

Riketta, M. (2002), “Attitudinal organizational commitment and job performance: a meta-analysis", Journal of Organizational Behavior, Vol. 23, pp. 257-66.

Rashid et al, (2003); The influence of corporate culture and organizational commitment on performance Journal of Management Development, Vol. 22 No. 8, 2003, pp. 708-728

Si, S. X., \& Cullen, J. B. (1998). Response categories and potential cultural bias: Effects of an explicit middle point in cross-cultural surveys. International Journal of Organizational Analysis, 6, 218.

Solinger Omar N., Woody van Olffen, \& Robert A. Roe (2008); Beyond the Three-Component Model of Organizational Commitment; Journal of Applied Psychology; 2008, Vol. 93, No. 1, pp, 70-83

Somers, M. \& Bimbaum, D. (2000), "Exploring the relationship between commitment profiles and work attitudes, employee withdrawal, and job performance", Public Personnel Management, Vol. 29 No. 3, pp. 353-64.

Shukla S. (2015); Conference paper; found at: https://www.researchgate.net/publication/299597986 on $14 / 10 / 2020$

Steijn, B. and Leisink, P. (2006), "Organizational commitment among Dutch public sector employees", International Review of Administrative Sciences, Vol. 72, pp. 187-201.

Sturgis, P., Roberts, C., Smith, P.: Middle alternatives revisited: how the neither/nor response acts as a way of saying “'I don't know'? Sociol. Methods Res. 43, 15-38 (2014). doi:10.1177/0049124112452527

Swaidan, Victoria R., (2018) "Strategies to Adjust for Response Bias in Clinical Trials: A Simulation Study".Graduate Theses andDissertations.https://scholarcommons.usf.edu/etd/7648

Tourangeau, R., Couper, M.P., Conrad, F.: (2004). Spacing, position, and order. interpretive heuristics for visual features of survey questions. Public Opin. Q. 68, 368-393 . doi:10.1093/poq/nfh035

Vandanbergghe, C. (1996). Assessing Organizational Commitment in a Belgian context. Evidence for threedimensional model. Applied Psychology: An international Review. Vol. 45, pp, 371-386

Weijters, B., Cabooter, E., Schillewaert, N.: (2010). The effect of rating scale format on response styles: the number of response categories and response category labels. Int. J. Res. Mark. 27, 236-247 . doi:10. 1016/j.ijresmar.2010.02.004

Wilson Mugizi,Fred E. K. Bakkabulindi\&Jude Ssempebwa (2016); Validity and Reliability of Allen and Meyer's (1990) Measure of EmployeeCommitment in the Context of Academic Staff in Universitiesin Uganda; Journal of Sociology and Education in Africa; Vol. 14 No. 1

Yousef, D.A. (2002), "Job satisfaction as a mediator of the relationship between role stressors and organizational commitment. A study from an Arabic perspective", Journal of Managerial Psychology, Vol. 17, pp. 250-66. 